\title{
Vertical intensity modulation for improved radiographic penetration and reduced exclusion zone
}

\author{
J. Bendahan* and W.G.J. Langeveld \\ Rapiscan Laboratories, Inc. 520 Almanor Avenue, \\ Sunnyvale, California 95129, USA \\ *jbendahan@rapiscanSystems.com \\ V. Bharadwaj ${ }^{\dagger}$, J. Amann, C. Limborg and Y. Nosochkov \\ SLAC, 2575 Sand Hill Road, \\ Menlo Park, California 94025,USA \\ †vinod@SLAC.Stanford.EDU
}

Published 1 September 2016

\begin{abstract}
In the present work, a method to direct the X-ray beam in real time to the desired locations in the cargo to increase penetration and reduce exclusion zone is presented. Cargo scanners employ high energy X-rays to produce radiographic images of the cargo. Most new scanners employ dualenergy to produce, in addition to attenuation maps, atomic number information in order to facilitate the detection of contraband. The electron beam producing the bremsstrahlung X-ray beam is usually directed approximately to the center of the container, concentrating the highest Xray intensity to that area. Other parts of the container are exposed to lower radiation levels due to the large drop-off of the bremsstrahlung radiation intensity as a function of angle, especially for high energies $(>6 \mathrm{MV})$. This results in lower penetration in these areas, requiring higher power sources that increase the dose and exclusion zone. The capability to modulate the X-ray source intensity on a pulse-by-pulse basis to deliver only as much radiation as required to the cargo has been reported previously. This method is, however, controlled by the most attenuating part of the inspected slice, resulting in excessive radiation to other areas of the cargo. A method to direct a dual-energy beam has been developed to provide a more precisely controlled level of required radiation to highly attenuating areas. The present method is based on steering the dual-energy electron beam using magnetic components on a pulse-to-pulse basis to a fixed location on the $\mathrm{X}$ ray production target, but incident at different angles so as to direct the maximum intensity of the produced bremsstrahlung to the desired locations. The details of the technique and subsystem and simulation results are presented.
\end{abstract}

Keywords: X-ray scanner; intensity modulation; penetration; low dose; small exclusion zone.

\section{Introduction}

Rapiscan Systems, Inc. has deployed Eagle R60 and R90 railcar scanners in Europe and the Middle East. The most advanced operational system, the Eagle R60, is located about 15 miles from the port of Rotterdam in the Netherlands. The system performs dualenergy scans of railcars moving at speeds up to $60 \mathrm{kph}$. Although its $\sim 35 \mathrm{~cm}$ steel

This is an Open Access article published by World Scientific Publishing Company. It is distributed under the terms of the Creative Commons Attribution 3.0 (CC-BY) License. Further distribution of this work is permitted, provided the original work is properly cited. 
penetration is suitable for most railcars, there is a need for additional penetration for some cargos (e.g. tankers and bulk cargo).

$\mathrm{X}$-ray radiography cargo scanners used for the detection of contraband require high energy X-ray sources. The higher the intensity at a specific source energy, the greater the amount of material the X-ray beam can penetrate. Usually, the X-ray source intensity of the scanner is set to the highest level allowable under a particular scenario, whether an accurate inspection of the cargo requires this intensity or not. Typically, the source intensity is set so that the dose does not exceed $0.5 \mu \mathrm{Sv}$ at the boundary of the exclusion zone, or, in the case of portal inspection systems, where trucks are driven through the inspection system, to keep the scattered radiation below a certain dose limit to the driver. Therefore, there are areas of the cargo that are inspected with higher radiation dose than desired and some areas with lower radiation dose, resulting in larger exclusion areas and/or lower penetration than desired.

A type of source called an IMAXS (Intensity Modulated Advanced X-Ray Source) has been reported previously. ${ }^{1,2,3}$ Here, the X-ray source intensity is adjusted based on the maximum attenuation of the previous slice. Electronics in the detector array detects the maximum attenuation within the slice and sends commands to the source to change the intensity to an appropriate value. Simulation results based on recorded cargo images have shown that the exclusion zone can be reduced by an average factor of 3 and/or the system penetration can be increased by a factor of approximately 2 compared with systems without IMAXS.

Most X-ray sources employ accelerated electrons that impinge on a high-Z target to produce bremsstrahlung radiation. Usually, the electron beam is directed to the vertical center of the object being scanned. This radiation is increasingly forward peaked in both $\mathrm{X}$-ray intensity and X-ray energy as a function of increasing electron energy, relative to the X-ray intensity and energy in the electron beam direction. In many cases, the highest density areas may be found in off-center angular locations, resulting in lower penetration of those areas. Therefore, there is a need for finer intensity adjustment for improved exclusion zone reduction, reduced dose to cargo, and higher penetration without increasing the average source intensity.

\section{X-Ray Transport Simulations}

The penetration of high energy X-ray systems is determined by scanning an ANSI 42.46 standard penetration phantom. In accordance with the standard, the phantom is placed at the center of a rail cargo container and tilted towards the source. The X-rays are measured with the system's array of X-ray detectors (see Fig. 1).

To determine the improvements in penetration employing various methods, Geant $4{ }^{4}$ simulations were performed with a model of the ANSI penetration standard. For penetration beyond about $35 \mathrm{~cm}$ of steel, Geant 4 simulations are very time consuming ( $>10$ hours with a 64 core AMD computer), with run time increasing exponentially for higher penetration. To speed up the simulations, modified variance reduction techniques of splitting, Russian roulette and energy biasing with post processing were implemented. 
These modifications enabled obtaining results for penetration beyond $60 \mathrm{~cm}$ in several hours with the same computer.

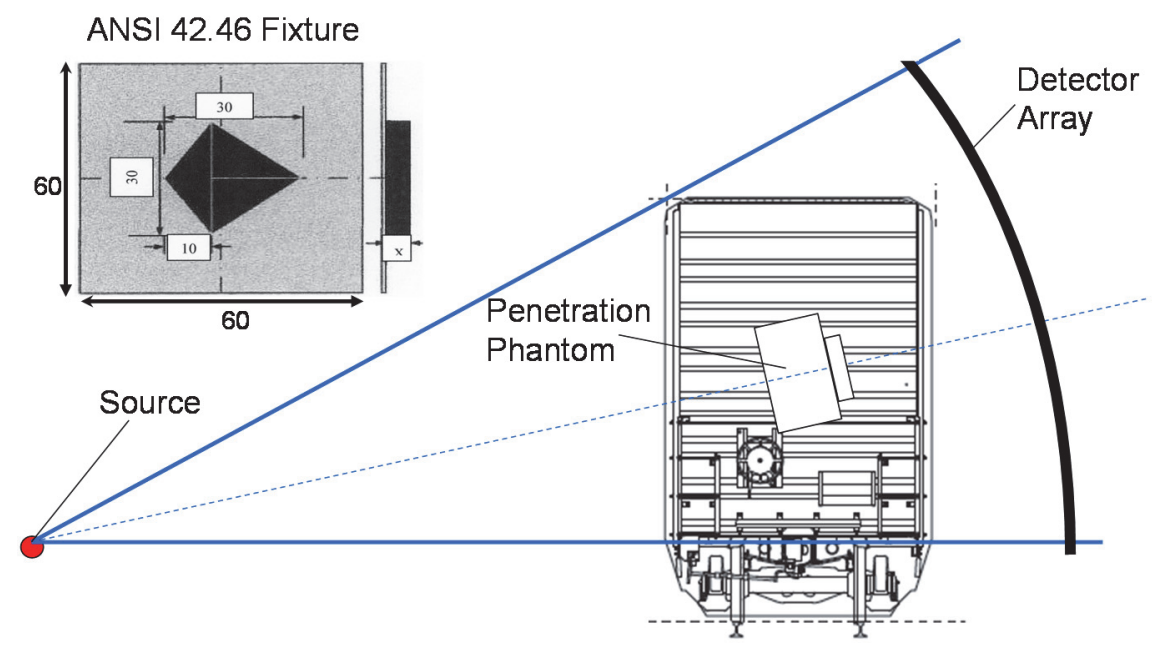

Fig. 1. Configuration used to simulate penetration. The ANSI 42.46 penetration phantom is shown in the upperleft corner.

Accelerated Geant4 simulations were performed with the model of the ANSI phantom for various system configurations and X-ray intensities at 9.5 MV. The Contrast-to-Noise ratio (CNR) was calculated for simulated radiographic images. Here we report the results for two angular positions: horizontal (Fig. 2, left) and beam center line (Fig. 2, right). The CNR is shown in the upper left corner. The penetration in the horizontal view is clearly lower compared to the center due to the lower X-ray intensity and lower average X-ray energy. This indicates that if the electron beam could be steered to the appropriate high attenuation location, the penetration would increase.
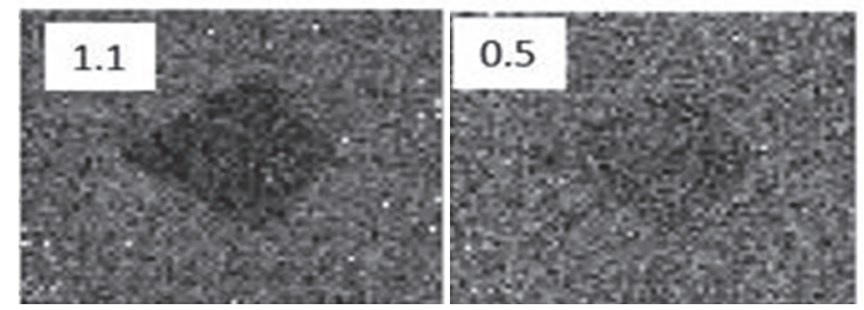

Fig. 2. Radiographic images of ANSI penetration phantom at horizontal position (left) and at beam center (right). The CNR is indicated at the top left of the images.

\section{Vertical Intensity Modulation (VIM)}

In existing X-ray scanners, a pulsed electron beam is directed to a fixed vertical location, producing a collimated fan beam of X-ray radiation with its highest intensity and average 
X-ray energy in the electron beam direction. This, however, does not necessarily illuminate the area requiring the highest radiation intensity: cargo can be very heterogeneous with high attenuation areas located at random locations. But even if prior information is available, it is difficult in such systems to move the electron beam direction in real time.

The approach presented here consists of directing the electron beam to the areas with highest attenuation in milliseconds or fractions thereof, based on the analysis of the previous image slice. Electron beam steering is achieved in this concept with a series of magnetic elements that direct the beam to a fixed point on the target but pointing in the desired direction. A single-energy version of this idea was presented in Ref. 5. The dualenergy system consists of a chicane, followed by a quadrupole, a kicker magnet and another quadrupole (see Fig. 3).

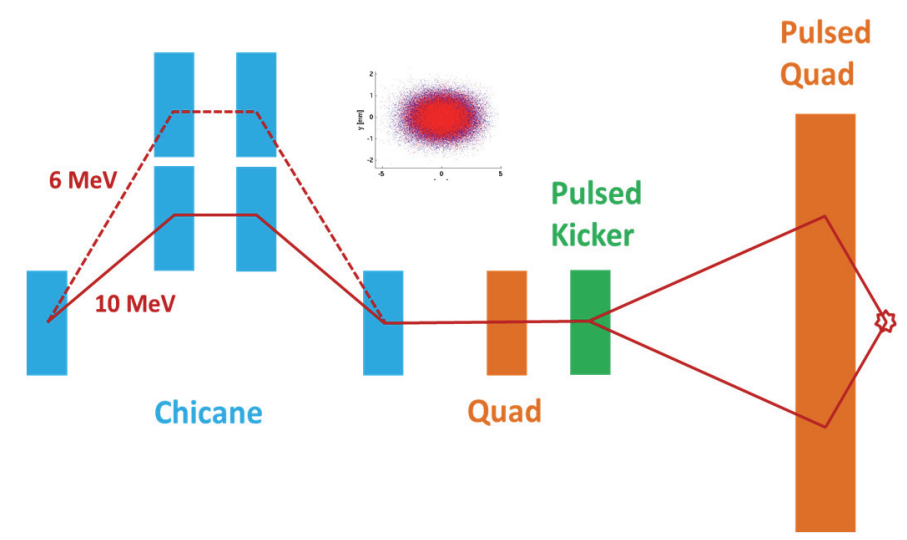

Fig. 3. Schematic of dual-energy electron beam transport system.

The chicane consists of six dipoles and two slits, which allow filtering the low-energy tails produced by most linacs. These low-energy electrons would hit the beam pipe and other locations of the target if not removed. During the electron transport through the chicane, the beam diverges and a quadrupole is required to refocus the electrons before continuing the transport.

The particle trajectories were simulated with the ASTRA code ${ }^{6}$ with and without the slits, which were adjusted to cut the low energy tails without affecting the primary beam, as shown in Fig. 4. The magnetic fields were set at $700 \mathrm{G}$ for each of the magnets. To determine the magnet design parameters, tracking was performed using four particles, two of them at $5.98 \mathrm{MeV}$ and two at $9.85 \mathrm{MeV}$. For each pair, one particle had no transverse offset $(\mathrm{x}=0 \mathrm{~mm})$ and the second one had an $\mathrm{x}=100 \mu \mathrm{m}$ offset. Two of the particles were separated by less than $100 \mu \mathrm{m}$ at $\mathrm{z}=1.3 \mathrm{~m}$ and less than $200 \mu \mathrm{m}$ at $\mathrm{z}=2 \mathrm{~m}$, which is acceptable. 

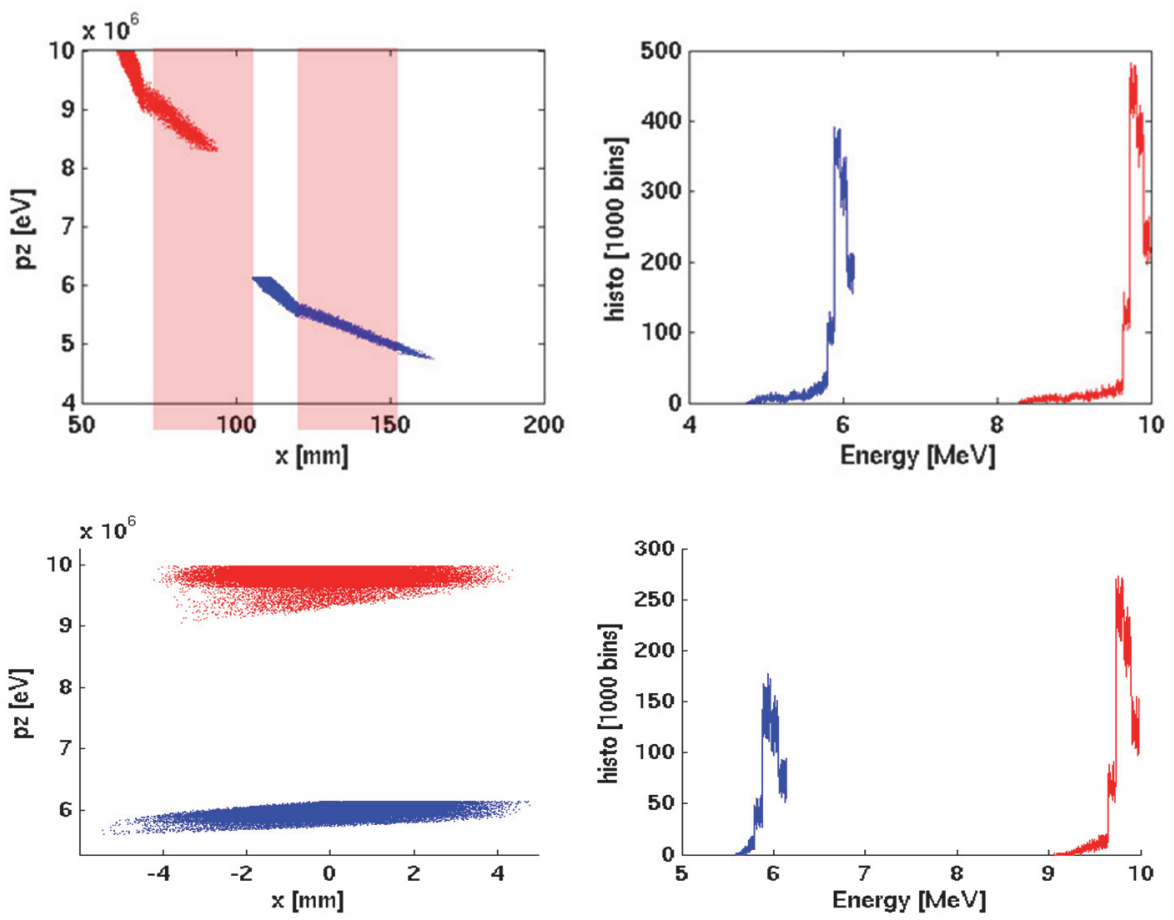

Fig. 4. Two-energy distributions at $\mathrm{z}=1.3 \mathrm{~m}$ within the chicane (top) and after the chicane (bottom). $\mathrm{X}-\mathrm{Y}$ are the tranverse direction, and $\mathrm{Z}$ is along the beam.

There are two options to compensate for the $\pm 0.5 \mathrm{~mm}$ beam position variation at the target (Fig. 4, lower left). The first option is to provide a minor adjustment of the quadrupole field gradient as a function of beam angle at the target. This adjustment, however, must be done at the $\sim 1 \mathrm{kHz}$ frequency. It was found that the maximum adjusted integrated quadrupole gradient is increased by $2.1 \%$ (to $\mathrm{B}^{\prime} \mathrm{L}=3.524 \mathrm{kG}$ ) compared to the constant gradient option.

The second option requires an octupole corrector magnet placed before the quadrupole to generate a nonlinear compensating effect on the vertical beam orbit $\left(\sim \mathrm{y}^{3}\right)$. In this case, the field strengths of neither the octupole nor the quadrupole depend on beam angle at the target, but they must be switched to either $6 \mathrm{MeV}$ or $10 \mathrm{MeV}$ values (proportional to the beam energy) on the $\sim 1 \mathrm{kHz}$ time scale. With proper parameters, the variation of beam center position at the target with the beam angle is canceled. An additional benefit of the octupole is that the horizontal beam size at large angles is reduced. This is due to the fact that the octupole, in this case, generates a feed-down horizontally focusing quadrupole effect on the beam orbit, which is proportional quadratically with the kicker angle. 


\section{$J$. Bendahan et al.}

The beam position at the target can be affected by quadrupole misalignment errors. MAD ${ }^{9}$ with a hard edge quadrupole model was used to determine the $\mathrm{x}$ and $\mathrm{y}$ alignment. In this case, the tolerances are independent of the beam angle at the target, except that the roll tolerance for the pulsed quadrupole gets looser at smaller beam angles (tolerance inversely proportional to angle). The pulsed quadrupole tolerances are independent of beam energy, while the fixed strength quadrupole tolerances are tighter for the $6 \mathrm{MeV}$ beam. Table 1 shows the tightest tolerances for the two beam energies and the range of angles. One could significantly loosen these tolerances by adding a weak dipole corrector (a few G.m) next to the fixed strength quadrupole. Such a corrector could compensate any static position error at the target.

Table 1. Magnet field strengths and beam parameters at target for the complete transport line and three angles at target: $-5^{\circ},-15^{\circ}$, and $-25^{\circ}$.

\begin{tabular}{|l|c|c|c|c|c|c|}
\hline \multicolumn{1}{|c|}{ Energy } & \multicolumn{3}{|c|}{$\mathbf{6 ~ M e V}$} & \multicolumn{3}{c|}{$\mathbf{1 0} \mathbf{~ M e V}$} \\
\hline \multicolumn{1}{|c|}{ y-angle } & $\mathbf{- 5}^{\circ}$ & $\mathbf{- 1 5 ^ { \circ }}$ & $\mathbf{- 2 5}^{\circ}$ & $\mathbf{- 5}^{\circ}$ & $\mathbf{- 1 5}^{\circ}$ & $\mathbf{- 2 5}^{\circ}$ \\
\hline Kicker BL (G·m) & 5.07 & 15.18 & 25.06 & 8.35 & 24.96 & 41.28 \\
\hline Pulsed quad B'L (kG) & 2.135 & 2.114 & 2.077 & 3.512 & 3.477 & 3.416 \\
\hline Fixed quad B'L (kG) & 0.41 & 0.41 & 0.41 & 0.41 & 0.41 & 0.41 \\
\hline Beam x-sigma (mm) & 2.19 & 2.19 & 2.20 & 2.19 & 2.19 & 2.19 \\
\hline Beam y-sigma (mm) & 0.53 & 0.87 & 1.35 & 0.20 & 0.44 & 0.73 \\
\hline x-position (mm) & -0.50 & -0.50 & -0.50 & -0.02 & -0.02 & -0.02 \\
\hline y-position (mm) & 0.00 & 0.00 & 0.00 & 0.00 & 0.00 & 0.00 \\
\hline x-angle (deg) & -0.14 & -0.14 & -0.14 & -0.004 & -0.004 & -0.004 \\
\hline
\end{tabular}

Fig. 5 shows the overall layout of the beam line from the output of the electron linac to the bremsstrahlung target. In this figure, the beam enters from the right, passes through the low energy beam tail cleanup chicane, then through a fixed quadrupole for controlling the beam-line optics and finally through the pulsed dipole/pulsed quadrupole for adjusting the angle of incidence at the X-ray production target.

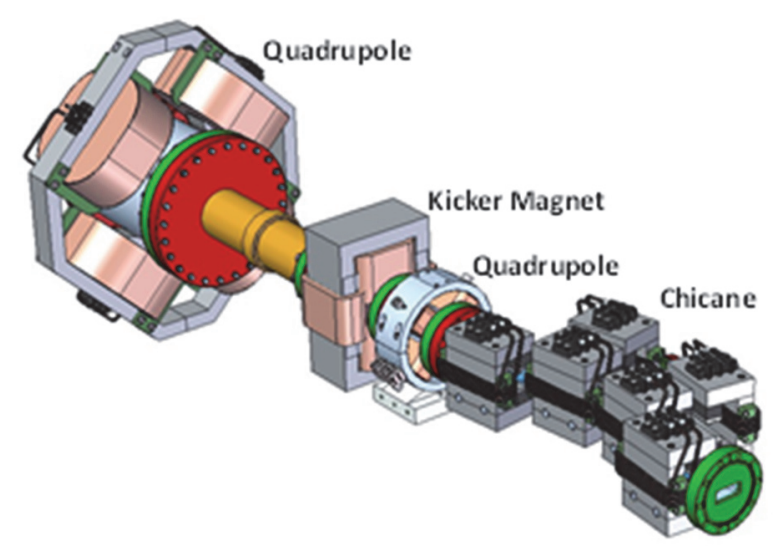

Fig. 5. Layout of the beam line. 


\section{Conclusions}

Extensive electron beam transport simulations were performed to design a beam line that would produce a beam with adjustable angles of incidence at the same point of an X-ray production target. This Vertical Intensity Modulation capability allows for improved radiographic penetration as the beam is directed to areas with high attenuation, and results in reduced exclusion zones.

\section{Acknowledgments}

This work has been partially supported by the US Department of Homeland Security, Domestic Nuclear Detection Office, under competitively awarded contract HSHQDC-14C-B0001. This support does not constitute an expressed or implied endorsement on the part of the Government.

\section{References}

1. W.G.J. Langeveld, W.A. Johnson, R.D. Owen and R.G. Schonberg, Intensity Modulated Advanced X-ray Source (IMAXS) for homeland security applications (I), IEEE Trans. Nucl. Sc. 56, 1288 (2009).

2. W.G.J. Langeveld, W.A. Johnson, R.D. Owen and R.G. Schonberg, Intensity Modulated Advanced X-ray Source (IMAXS) for homeland security applications (II), AIP Conf. Proc. 1099, 628 (2009).

3. W.G.J. Langeveld, C. Brown, P.A. Christensen, C. Condron, M. Hernandez, M. Ingle, W.A. Johnson, R.D. Owen, R. Ross and R.G. Schonberg, Performance characteristics of an Intensity Modulated Advanced X-ray Source (IMAXS) for homeland security applications, AIP Conf. Proc. 1336, 705 (2011).

4. Geant4 Collaboration, Nucl. Instr. Meth. A 506, 250 (2003), IEEE Trans. Nucl. Sc. 53 No. 1, 270 (2006).

5. J. Clayton, D. Shedlock, W.G.J. Langeveld, V. Bharadwaj, and Y. Nosochkov, Physics Procedia 66, 249 (2015).

6. ASTRA: http://www.desy.de/ mpyflo/.

7. OPERA: Cobham Technical Services, Vector Fields Inc., http://operafea.com.

8. M. Borland, elegant: A Flexible SDDS-Compliant Code for Accelerator Simulation, Advanced Photon Source LS-287, September 2000.

9. H. Grote and F. Ch. Iselin, The MAD Program (Methodological Accelerator Design), User's Reference Manual, CERN/SL/90-13(AP), April 29, 1996. 